\title{
Mejora de un modelo de representación de materiales para su uso en edición intuitiva de apariencia
}

\author{
Sandra Malpica, Miguel Barrio, Diego Gutiérrez, Ana Serrano, Belén Masiá \\ Graphics and Imaging Lab \\ Instituto de Investigación en Ingeniería de Aragón (I3A) \\ Universidad de Zaragoza, Mariano Esquillor s/n, 50018, Zaragoza, Spain. \\ Tel. +34-976762707, e-mail: $\underline{670607 @ u n i z a r . e s}$
}

\section{Resumen}

Durante los últimos años se ha producido un gran avance en las técnicas de captura de materiales. Sin embargo, editar de forma intuitiva materiales sigue siendo un reto. Este trabajo se basa en un método existente y consigue ampliar el rango de apariencia que se puede obtener durante la edición.

\section{Introducción}

El aumento de eficiencia y precisión de las técnicas de medición de apariencia de materiales (por ejemplo, [1] o [2]) ha llevado a un mayor número de bases de datos públicas disponibles y técnicas de modelado. Sin embargo, la edición de apariencia de materiales sigue siendo un reto debido a varias razones, entre ellas la desconexión entre la representación de los datos y el entendimiento humano y la complejidad y alta dimensionalidad de los datos medidos que dificultan la utilización de parámetros de edición intuitivos. Sin embargo, el aumento de disponibilidad de información ha ayudado a la construcción de nuevas técnicas de edición para los datos capturados que han conseguido sobrepasar las limitaciones mencionadas a la hora de editar la apariencia de materiales de forma intuitiva. Sobre este tipo de trabajos destaca el de Serrano et al. [3] debido a su facilidad de utilización para usuarios inexpertos.

Este trabajo se basa en la mejora del procedimiento utilizado por Serrano et al. para la edición intuitiva de materiales. En su trabajo, Serrano et al. se basan en una representación de BRDFs que consiste en su descomposición en componentes principales mediante (PCA) para reducir su dimensionalidad. Una vez han obtenido los componentes principales de las BRDFs, entrenan una serie de funcionales que los relacionan con un conjunto de atributos intuitivos. De esta forma una modificación del valor de un atributo en una BRDF se traduce en una modificación de sus componentes principales y por tanto de su apariencia.

Para entrenar su base PCA, Serrano et al. utilizan la base de datos de MERL, un conjunto de 100 BRDFs de distintos tipos de materiales capturadas por Matusik et al. [4]. Esta base tiene un mayor número de muestras de materiales especulares, por lo que está sesgada. Pese a aplicar un filtro logarítmico sobre los datos al igual que Nielsen et al. en su trabajo [5] para reducir el efecto de los picos especulares sobre la base PCA, el sesgo de las muestras iniciales implica que ciertos materiales muy poco especulares (o muy difusos) no se representen correctamente debido a la aparición de halos o círculos oscuros en ciertas zonas del material. Para superar este problema, en este trabajo se propone la creación de una nueva base PCA entrenada sólo con las muestras difusas de la base de datos de MERL, a la que llamaremos base soft PCA. Una vez obtenida la nueva base, se propone una nueva medida de especularidad para materiales capturados que nos permite definir una frontera entre la nueva representación y la original de Serrano et al. (a la que llamaremos base full PCA) y una técnica de navegación entre ambas representaciones para evitar la aparición de artefactos en los materiales difusos. Este trabajo ha sido aceptado en la Conferencia Española de Informática Gráfica de 2017.

\section{Creación del nuevo sistema}

Inicialmente se seleccionan manualmente las 40 BRDFs más difusas de la base de datos de MERL para entrenar una nueva base PCA. En la Figura 1 se puede comprobar que la base de soft PCA puede representar correctamente los materiales difusos. La Figura 2 muestra una comparación entre ambas bases. Una vez obtenida la nueva representación, se utilizan los mismos atributos del trabajo de Serrano et al. y se entrenan funcionales que los relacionan con los componentes de la base de soft $P C A$. En la figura 3 se puede ver, para una misma 
zona de los distintos espacios de las bases PCA, cómo el valor de los atributos varía de forma diferente. Debido al tipo de datos con los que ha sido entrenada la base

soft PCA, nuestra base no es capaz de representar de forma correcta los materiales especulares. Por ello, necesitamos una medida de la especularidad que nos permita cambiar de representación cuando sea necesario y un método para navegar entre ambas representaciones durante la edición.

Para ello nos inspiramos en las direcciones óptimas de captura de BRDFs con pocas muestras calculadas por Nielsen [1] y las utilizamos para crear una nueva medida de especularidad válida para cualquier material capturado. Comprobamos que todos los materiales difusos presentan una medida de especularidad inferior a 0.4, y utilizamos este límite para entrenar Support Vector Machines que definan de forma automática una frontera en los dos espacios PCA. Utilizamos esta frontera para elegir el punto en el que cambiar de representación. Como técnica para ello, utilizamos dos redes neuronales de base radial que relacionan los cinco componentes de cada BRDF en la base de full PCA con sus correspondientes en la base de soft PCA y viceversa. Por último, integramos el nuevo sistema de edición con dos representaciones de BRDFs en un plugin interactivo para facilitar las ediciones al usuario.

\section{Conclusiones y resultados}

Con nuestro nuevo sistema de dos representaciones y la nueva medida de especularidad propuesta conseguimos superar las limitaciones del trabajo de Serrano et al. Gracias a ello, ampliamos el espacio de posibles ediciones de materiales permitiendo al usuario obtener materiales difusos que se representan correctamente. Además, mantenemos el sistema intuitivo de Serrano et al. que permite ediciones sencillas a usuarios inexpertos. La Figura 4 muestra una edición realizada con nuestro plugin.

\section{REFERENCIAS}

[1]. NIELSEN J. B., JENSEN H. W., RAMAMOORTHI R.: On Optimal, Minimal BRDF Sampling for Reflectance Acquisition. ACM Trans. Graph. 34, 6 (Nov. 2015).

[2]. AITTALA M., WEYRICH T., LEHTINEN J.: Twoshot SVBRDF capture for stationary materials. ACM Trans. Graph. 34, 4 (July 2015), 110:1-13.
[3]. SERRANO A., GUTIERREZ D., MYSZKOWSKI K., SEIDEL H.-P., MASIA B.: An intuitive control space for material appearance. ACM Transactions on Graphics (SIGGRAPH ASIA 2016) 35, 6 (2016).

[4]. MATUSIK W., PFISTER H., BRAND M., MCMILLAN L.: A data-driven reflectance model. ACM Trans. Graph. 22, 3 (July 2003),759-769.

[5]. NIELSEN J. B., JENSEN H. W., RAMAMOORTHI R.: On Optimal, Minimal BRDF Sampling for Reflectance Acquisition. ACM Trans. Graph. 34, 6 (Nov. 2015).

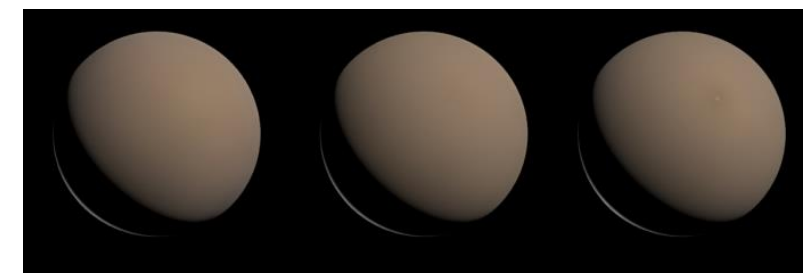

Figura 1: A la izquierda se muestra la BRDF original de la base de datos de MERL. En el centro la reconstrucción a partir de los componentes de la base soft PCA y a la derecha la reconstrucción de la base full PCA.
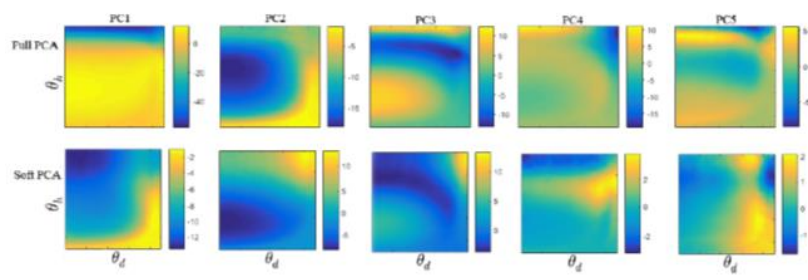

Figura 2: Cortes bidimensionales de los eigenvectores de los cinco componentes principales utilizados en la base full PCA (arriba) y en la de soft PCA (abajo). El primer componente principal (columna izquierda) representa la información del pico especular en la base full PCA.
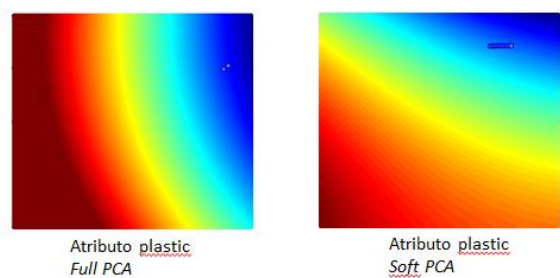

Figura 3: cortes bidimensionales del espacio 5D de la base de full PCA (izquierda) y de la de soft PCA (derecha) en los que se puede ver la variación del valor del atributo plastic.

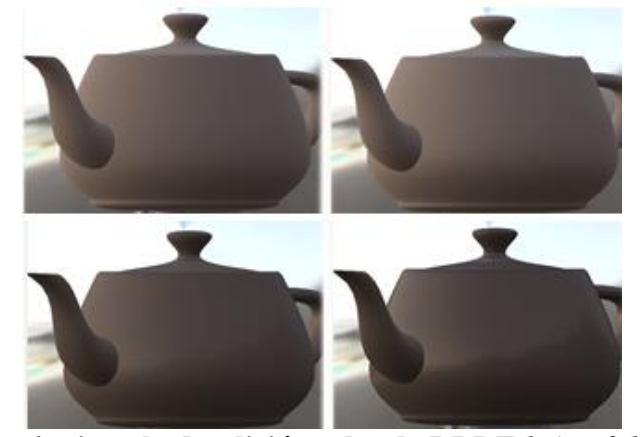

Figura 4: ejemplo de edición sobre la BRDF beige-fabric (izquierda) aumentando el valor del atributo metálico. Se produce un paso de la base soft PCA a la de full PCA.

Revista "Jornada de Jóvenes Investigadores del I3A", vol. 5 (Actas de la VI Jornada de Jóvenes Investigadores del I3A - 2 de junio de 2017). ISSN 2341-4790. 